PROCEEDINGS OF THE

AMERICAN MATHEMATICAL SOCIETY

Volume 135, Number 8, August 2007, Pages 2637-2647

S 0002-9939(07)08750-3

Article electronically published on March 21, 2007

\title{
PERIODIC SEGMENT IMPLIES INFINITELY MANY PERIODIC SOLUTIONS
}

\author{
WACŁAW MARZANTOWICZ AND KLAUDIUSZ WÓJCIK \\ (Communicated by Carmen C. Chicone)
}

\begin{abstract}
In this note we show that the existence of a periodic segment for a non-autonomous ODE with periodic coefficients implies the existence of infinitely many periodic solutions inside this segment provided that a sequence of Lefschetz numbers of iterations of an associated map is not constant. In the case when this sequence is bounded we have to impose a geometric condition on the segment to get solutions by use of symbolic dynamics.
\end{abstract}

\section{Periodic Segments}

Let $v: \mathbb{R} \times M \rightarrow T M$ be a smooth (i.e. $C^{k}, k \geq 1$ ) time-dependent vector-field on a manifold $M$ of dimension $\operatorname{dim} M=m$. We consider the non-autonomous equation

$$
\dot{x}=v(t, x)
$$

with $T$-periodic coefficients in $t$, i.e. there is $T>0$ such that $v(t+T, x)=v(t, x)$ for every $(t, x)$. Every such equation generates a $T$-periodic local process $\Phi$ on $M$ such that for $t_{0} \in \mathbb{R}$ and $x_{0} \in M$ the map

$$
\left(\alpha_{\left(t_{0}, x_{0}\right)}, \omega_{\left(t_{0}, x_{0}\right)}\right) \ni t \mapsto \Phi_{\left(t_{0}, t\right)}\left(x_{0}\right) \in M
$$

is the maximal solution of the initial value problem $\dot{x}=v(t, x), \quad x\left(t_{0}\right)=x_{0}$.

The map $\Phi_{(0, T)}$ is called the Poincaré map. Recall that if the vector field is of a class $C^{k}, k \geq 1$, then the Poincare map is of the same class. We mention that if $\phi$ is a local flow on the extended phase space $\mathbb{R} \times M$ generated by the system of equations

$$
\dot{x}=v(t, x), \quad \dot{t}=1,
$$

then

$$
\phi\left(\left(t_{0}, x_{0}\right), t\right)=\left(t_{0}+t, \Phi_{\left(t_{0}, t\right)}\left(x_{0}\right)\right) .
$$

One of the basic tools of geometrical theory of periodic solutions is the notion of a periodic segment introduced in [S1]. Let $W \subset \mathbb{R} \times M$. Define the exit set of $W$ as

$$
W^{-}:=\left\{(\tau, x) \in W: \phi((\tau, x),[0, t]) \not \subset W \forall t \in\left(0, \omega_{(\tau, x)}\right)\right\} .
$$

Received by the editors January 2, 2006 and, in revised form, April 7, 2006.

2000 Mathematics Subject Classification. Primary 54H25, 37B35, 37B55.

Key words and phrases. Periodic solution, periodic segment, Lefschetz number, fixed point index.

The first author's research was supported by KBN grant 2P03A 03929.

(C)2007 American Mathematical Society Reverts to public domain 28 years from publication 
We call $W$ a block for $\phi$ if $W, W^{-}$are compact.

In order to define a periodic segment in the extended phase space we introduce the following notation: by $\pi_{1}$ and $\pi_{2}$ we denote the projections of $\mathbb{R} \times M$ onto $\mathbb{R}$ and, respectively, $M$, and if $Z$ is a subset of $\mathbb{R} \times M$ and $t \in \mathbb{R},\left[t_{1}, t_{2}\right] \subset \mathbb{R}$, then we put

$$
Z_{t}:=\{z \in M:(t, z) \in Z\}, \quad Z_{\left[t_{1}, t_{2}\right]}=Z \cap\left(\left[t_{1}, t_{2}\right] \times X\right) .
$$

A set $W \subset[0, T] \times M$ is called a periodic segment over $[0, T]$ (for the equation (10) if it is a block with respect to $\phi$ such that the following conditions hold:

(a) there exists a compact subset $W^{--}$of $W^{-}$(called the essential exit set) such that

$$
W^{-}=W^{--} \cup\{T\} \times W_{T}, \quad W^{-} \cap([0, T) \times M) \subset W^{--},
$$

(b) there exists a homeomorphism $h:[0, T] \times W_{0} \rightarrow W$ such that $\pi_{1} \circ h=\pi_{1}$ and

$$
h\left([0, T] \times W_{0}^{--}\right)=W^{--},
$$

(c) $\left(W_{0}, W_{0}^{--}\right)=\left(W_{T}, W_{T}^{--}\right)$are ENR's (the Euclidean Neighborhood Retracts).

Using a homeomorphism $h$ satisfying (b) we define a monodromy homeomorphism

$$
\mathbf{m}:\left(W_{0}, W_{0}^{--}\right) \rightarrow\left(W_{0}, W_{0}^{--}\right) \quad \text { by } \quad \mathbf{m}(x)=\pi_{2} h\left(T, \pi_{2} h^{-1}(0, x)\right) .
$$

It can be proved that a different choice of the homeomorphism satisfying (b) provides the monodromy homoeomorphism homotopic to $\mathbf{m}$ (cf. [S1]). It follows, in particular, that the isomorphism in singular homologies (with coefficients in $\mathbb{Q}$ )

$$
\mu_{W}:=H(\mathbf{m}): H\left(W_{0}, W_{0}^{--}\right) \rightarrow H\left(W_{0}, W_{0}^{--}\right)
$$

is an invariant of the segment $W$.

Since $W_{0}, W_{0}^{--}$are compact ENR's, $H\left(W_{0}, W_{0}^{--}\right)$is of finite type and the Lefschetz number

$$
\Lambda\left(\mu_{W}\right)=\sum_{i \geq 0}(-1)^{i} \text { trace } H_{i}(\mathbf{m})
$$

is correctly defined.

In the sequel we will use the following theorem due to Srzednicki ([S1], and also [SWZ]).

Theorem 1.1. Let $W$ be a periodic segment over $[0, T]$. Then the set

$$
U=U_{W}:=\left\{x \in W_{0}: \Phi_{(0, t)}(x) \in W_{t} \backslash W_{t}^{--} \forall t \in[0, T]\right\}
$$

is open in $W_{0}$ and the set of fixed points of the restriction $\left.\Phi_{(0, T)}\right|_{U}: U \rightarrow W_{0}$ is compact. Moreover, $\operatorname{ind}\left(\left.\Phi_{(0, T)}\right|_{U}\right)=\Lambda\left(\mu_{W}\right)$. In particular, if $\Lambda\left(\mu_{W}\right) \neq 0$, then $\Phi_{(0, T)}$ has a fixed point in $W_{0}$.

\section{MAin RESUlts}

Let $W$ be a periodic segment over $[0, T]$ for a $T$-periodic local process $\Phi$. From now on we assume that our vector field, thus also $\Phi$, is of the class $C^{k}, k \geq 1$. We put

$$
\Lambda_{n}=\Lambda\left(\mu_{W}^{n}\right), \quad n \geq 0,
$$


where $\mu_{W}^{n}=\underbrace{\mu_{W} \circ \ldots \circ \mu_{W}}_{n}, \quad \mu_{W}^{0}=\operatorname{id}_{H\left(W_{0}, W_{0}^{-}\right)}$, and we define the dual sequence $\left\{\Lambda_{n}^{*}\right\}$ of $\left\{\Lambda_{n}\right\}$ by

$$
\Lambda_{n}^{*}=(-1)^{n}\left(\sum_{l=0}^{n}(-1)^{l}\left(\begin{array}{c}
n \\
l
\end{array}\right) \Lambda_{l}\right), \quad n \geq 0 .
$$

Theorem 2.1. Assume that $W$ is a periodic segment for the local process generated by (11), and the sequence $\left\{\Lambda_{n}\right\}$ is unbounded. Then there exists infinitely many periodic solutions of (1) in $W$.

Proof. This is essentially the Shub-Sullivan theorem (see [ShSu, and also [JM] for generalizations related topics). Suppose that the set of periodic points $\Phi_{(0, T)}$ is finite and consists of $m$ disjoint orbits

$$
x_{1}^{1}, \ldots, x_{i_{1}}^{1}, x_{1}^{2}, \ldots, x_{i_{2}}^{2}, \ldots, x_{1}^{s}, \ldots, x_{i_{s}}^{s} .
$$

Let $W^{n}$ be a periodic segment over [0,nT] obtained by gluing together $n$ translated copies of $W$. It follows that $\mu_{W^{n}}=\mu_{W}^{n}$. By Theorem 1.1 the excision and the additivity properties of the fixed point index

$$
\sum_{j=1}^{s} \operatorname{Ind}\left(\Phi_{(0, T) \mid U_{W^{n} \cap V_{j}}^{n}}\right)=\Lambda\left(\mu_{W}^{n}\right)
$$

where $V_{j}$ is an open set containing $\left\{x_{1}^{j}, \ldots, x_{i_{j}}^{j}\right\}$ and disjoint with the other orbits. Since $v$ is $C^{k}$, so $\Phi$ is $C^{k}$ and by the Shub and Sullivan theorem the left hand side is bounded, which leads to a contradiction.

Observe that in the case when $\left\{\Lambda_{n}\right\}$ is unbounded the existence of infinitely many periodic solutions is a consequence of a general theorem on periodic points of smooth maps. If the sequence of Lefschetz numbers of a smooth map is bounded, then the set of periodic points can be finite, e.g. empty. Similarly, a periodic equation (11) with a periodic segment and $\left\{\Lambda_{n}\right\}$ bounded has finitely many periodic solutions in general.

Example 2.2. The planar system $\dot{z}=\bar{z}$ for each $T>0$ posseses a periodic segment $W$ with the sequence $\left\{\Lambda_{n}\right\}$ constant equal to -1 and has just one trivial bounded solution. Consider the following planar non-autonomous equation of the variable $z \in \mathbb{C}:$

$$
\dot{z}=e^{i t} \bar{z}^{k} .
$$

The right-hand side of equation (44) is $T=2 \pi$ periodic. One can check that (4) has only the trivial periodic solution, there exists a $T$-periodic segment $W$, and $\Lambda_{n}$ is $k+1$-periodic with

$$
\Lambda_{1}=\ldots=\Lambda_{k}=1, \quad \Lambda_{0}=\Lambda_{k+1}=-k .
$$

In particular $\left\{\Lambda_{n}\right\}$ is non-constant.

Remark 2.3. The case when $\left\{\Lambda_{n}\right\}$ is bounded seems to be typical. In all examples known to the authors a constructed periodic segment $W$ and corresponding map $\mu$ lead to a bounded sequence $\left\{\Lambda_{n}\right\}$, thus are periodic by Lemma 2.6 and Theorem 2.7. We conjecture that the sequence $\left\{\Lambda_{n}\right\}$ is always bounded provided that a periodic segment exists. 
In case $\left\{\Lambda_{n}\right\}$ is bounded, to establish the existence of periodic solutions of equation (11) we have to impose additional geometric properties on the periodic segment $W$ introduced in [SW].

Assume that $Z, W$ are two periodic segments over $[0, T]$ with $\Lambda_{n}=\Lambda\left(\mu_{W}^{n}\right)$, and moreover the following two conditions hold:

$$
\begin{gathered}
Z \subset W, \quad Z_{0}=W_{0}, \quad Z_{0}^{--}=W_{0}^{--}, \\
\mu_{Z}=\operatorname{id}_{H\left(W_{0}, W_{0}^{-}\right)} .
\end{gathered}
$$

We define

$$
I:=\bigcap_{n=-\infty}^{\infty}\left\{x \in W_{0}: \Phi_{(0, t+n T)}(x) \in W_{t} \forall t \in[0, T]\right\},
$$

i.e. the set of all points in $W_{0}$ whose full trajectories are contained in the bigger segment $W$. It follows that $I$ is compact and invariant for the Poincaré map. For $x \in I$ we encode a trajectory of $x$ by the sequence $g(x) \in \Sigma_{2}=\{0,1\}^{\mathbb{Z}}$ by the following rule:

- if over the time interval $[i T,(i+1) T]$ the trajectory of $x$ is contained in $Z$, then $g(x)_{i}=0$,

- if $\Phi_{(0, T)}^{i}(x)$ leaves $Z$ in time less than $T$, then $g(x)_{i}=1$.

It follows that the map $g: I \rightarrow \Sigma_{2}$ is continuous and

$$
\sigma \circ g=g \circ \Phi_{(0, T)},
$$

where $\sigma: \Sigma_{2} \rightarrow \Sigma_{2}$ is a shift map.

Theorem 2.4. Assume that periodic segments $Z, W$ satisfy (15)-(6) and $\left\{\Lambda_{n}\right\}$ is bounded and non-constant. Then $g$ is surjective, i.e., the Poincaré map $\Phi_{(0, T)}$ is semi-conjugated to the shift $\sigma$ in I. Moreover, there exists an infinite set $S \subset \mathbb{N}$ with the property that for each $k \in S$ and each n-periodic sequence $c \in \Sigma_{2}$ such that the symbol 1 appears $k$ times in $c$, there exists $x \in g^{-1}(c)$ and $\Phi_{(0, T)}^{n}(x)=x$.

Proof. We separated an algebraic part of the proof and exposed it below. If $\left\{\Lambda_{n}\right\}$ is bounded, then from Proposition 2.9 it follows that there exists an infinite set $S \subset \mathbb{N}$ such that for each $k \in S, \Lambda_{k}^{*} \neq 0$. Fix $k \in S$. Let $c=\left(c_{0}, \ldots, c_{n-1}\right) \in \Sigma_{2}$ be $n$-periodic such that the symbol 1 appears exactly $k$-times in the sequence $c$. Let

$$
F[c]=\left\{x \in g^{-1}(c): \Phi_{(0, T)}^{n}(x)=x\right\} \subset I
$$

be the set that consists of all fixed points for the $n$-th iteration of the Poincaré map $\Phi_{(0, T)}^{n}$ whose trajectories are coded by the sequence $c$. One can prove (compare Th. 2 in $[\mathrm{W}])$ that the fixed point index $\operatorname{ind}\left(\Phi_{(0, T)}^{n}, F[c]\right)$ is well-defined and

$$
\operatorname{ind}\left(\Phi_{(0, T)}^{n}, F[c]\right)=\Lambda_{k}^{*} \neq 0,
$$

so $F[c] \neq \emptyset$. In particular, $c \in g(I)$. One can easily check that the set of all $n$-periodic sequences $c$ with $k \in S$ symbols 1 is dense in $\Sigma_{2}$, so $g$ is surjective.

Remark 2.5. Note that the assumption that the sequence $\left\{\Lambda_{n}\right\}$ is not constant is essential. Otherwise one can take $Z=W$ as being an isolating block for the origin in the planar system $\dot{z}=\bar{z}$. Then $\left\{\Lambda_{n}\right\}$ is a constant equal to -1 and the system for each $T>0$ has just one $T$-periodic solution. 
We say that a sequence $\Lambda_{n}$ of integers satisfies the Dold relations, or Dold congruences, if

$$
\forall n \in \mathbb{N} \quad \sum_{d \mid n} \mu(n / d) \Lambda_{d} \cong 0 \quad \bmod n,
$$

where $\mu: \mathbb{N} \rightarrow\{-1,0,1\}$ is the Möbius function (cf. [D], JM]).

Lemma 2.6. For each periodic segment $W$ the sequences $\left\{\Lambda_{n}\right\},\left\{\Lambda_{n}^{*}\right\}$ satisfy Dold's relations.

Proof. $\Lambda_{n}=L\left(\mu^{n}\right)$ is the Lefschetz number of a map of the pair $\left(W_{0}, W_{0}^{--}\right)$with finitely generated homology spaces. Then the first part of the statement follows from the corresponding Dold congruences for iterations of an integral matrix $A$ (cf. MP):

$$
\forall \quad n \in \mathbb{N} \quad \sum_{d \mid n} \mu(n / d) \operatorname{tr} A^{d} \cong 0 \quad \bmod n .
$$

To prove the second part of the statement observe that

$$
\Lambda_{n}^{*}=\sum_{j=0}^{n}\left(\begin{array}{l}
n \\
j
\end{array}\right) \Lambda_{j}(-1)^{n-j}=\sum_{j=0}^{n}\left(\begin{array}{l}
n \\
j
\end{array}\right)\left(\operatorname{tr} H_{e v}^{j}(\mathbf{m})-\operatorname{tr} H_{o d}^{j}(\mathbf{m})\right)(-1)^{n-j},
$$

where $H_{e v}(\mathbf{m})$, correspondingly $H_{o d}(\mathbf{m})$ is the induced map on the even dimensional, respectively odd dimensional homology spaces. Consequently

$$
\Lambda_{n}^{*}=\operatorname{tr}\left(H_{e v}(\mathbf{m})-\mathrm{I}\right)^{n}-\operatorname{tr}\left(H_{o d}(\mathbf{m})-\mathrm{I}\right)^{n},
$$

and the statement follows from (11) applied to the integral matrices $H_{e v}(\mathbf{m})-\mathrm{I}$ and $H_{o d}(\mathbf{m})$ - I respectively.

Suppose now that the sequence $\Lambda_{n}$ is bounded. Then we have the following algebraic characterization (cf. $[\mathrm{BB}$, also $[\mathrm{JM}]$ for another exposition).

Theorem 2.7. Suppose that $\Lambda_{n}$ is a sequence of integers satisfying the Dold relations. Then the following two conditions are equivalent:

i) The sequence is bounded.

ii) There exists a natural number $l>1$ and integers $a_{d}$ for all $d \mid l$ such that

$$
\Lambda_{n}=\sum_{d \mid l} a_{d} \sum_{j=1}^{d}\left(\epsilon_{d}\right)^{j n}, \quad n=1,2, \ldots,
$$

where $\left(\epsilon_{d}\right)^{d}=1$ is a primitive root of unity. In particular $\left\{\Lambda_{n}\right\}$ is periodic with period $l$.

Remark 2.8. Observe first that $l>1$ if $\left\{\Lambda_{n}\right\}$ is not a constant sequence. Otherwise $\Lambda_{n}=a_{1}\left(\epsilon_{1}=1\right)^{n}=a_{1}$. Observe next that if for the same reason $\left\{\Lambda_{n}\right\}$ is not the constant sequence, then there exists a coefficient $a_{d}, d>l, d \mid l$, such that $a_{d} \neq 0$.

As a consequence of Theorem 2.7 we have the following characterization of the dual sequence $\left\{\Lambda_{n}^{*}\right\}$.

Proposition 2.9. Assume that $\left\{\Lambda_{n}\right\}$ is bounded but not the constant sequence. Then $\left\{\Lambda_{n}^{*}\right\}$ is unbounded. Moreover there exists $k_{0}$ such that $\Lambda_{2 l k}^{*} \neq 0$ for $k>k_{0}$. Furthermore there exists $\rho>1$ such that $\lim _{k \rightarrow \infty} \frac{\Lambda_{2 l k}^{*}}{\rho^{2 l k}}=a \neq 0$. In particular there exist infinitely many primes $p$ for which $\Lambda_{2 l p}^{*} \neq 0$. 
Proof. By the definition

$$
\Lambda_{n}^{*}=\sum_{j=0}^{n}\left(\begin{array}{l}
n \\
j
\end{array}\right) \Lambda_{j}(-1)^{n-j} .
$$

Substituting the expression for $\Lambda_{n}$ given in Theorem 2.7 we have

$$
\begin{gathered}
\Lambda_{n}^{*}=\sum_{j=0}^{n}\left(\begin{array}{c}
n \\
j
\end{array}\right) \Lambda_{j}(-1)^{n-j}=\sum_{j=0}^{n}\left(\begin{array}{c}
n \\
j
\end{array}\right)(-1)^{n-j}\left(\sum_{d \mid l} a_{d} \sum_{i=1}^{d}\left(\epsilon_{d}^{i}\right)^{j}\right) \\
=\sum_{d \mid l} a_{d} \sum_{i=1}^{d}\left(\sum_{j=0}^{n}\left(\begin{array}{c}
n \\
j
\end{array}\right)\left(\epsilon_{d}^{i}\right)^{j}(-1)^{n-j}\right)=\sum_{d \mid l} a_{d} \sum_{i=1}^{d}\left(\epsilon_{d}^{i}-1\right)^{n} .
\end{gathered}
$$

Put $\lambda_{d, i}:=\epsilon_{d}^{i}-1$. Recall that $-1=\exp (\pi \imath)$, and consequently each $\lambda_{d, i}$ is equal to $\exp \left(\frac{2 \pi \imath i}{d}\right)+\exp (\pi \imath)$. By direct calculations we get

$$
\lambda_{d, i}=\rho_{d, i} \cdot \exp \left(\frac{2 \pi \imath}{d}\left(i+\frac{d}{2}\right)\right),
$$

where $\rho_{d, i}=\sqrt{2\left(1-\cos \left(\frac{2 \pi i}{d}\right)\right)}$. Note that $\rho_{d, j} \leq 2$, and for a given $d$ this maximum value of is achieved when

$$
\left\{\begin{array}{l}
d \text { is even and } i=\frac{d}{2}, \\
d \text { is odd and } i=\left[\frac{d}{2}\right], \text { or } i=\left[\frac{i}{2}\right]+1,
\end{array}\right.
$$

where $[x]$ denotes the integral part of a real number $x$.

Let $2 \geq \rho_{1} \geq \ldots \geq \rho_{r} \geq 0$ be all the radii appearing in (12), i.e. for every $1 \leq j \leq r$ there exists $(d, i), d \mid l$ and $1 \leq i \leq d$, such that $\rho_{j}=\rho_{d, i}$. Taking $n=2 l k, k \in \mathbb{N}$, we write our sum as

$$
\sum_{d \mid l} a_{d} \sum_{i=1}^{d} \rho_{d, i}^{n}=\sum_{j=1}^{r}\left(\sum_{(d, i) \in R_{j}} a_{d}\right) \rho_{j}^{n}=\sum_{j=1}^{r} A_{j} \rho_{j}^{n},
$$

where $R_{j}:=\left\{(d, i): \rho_{d, i}=\rho_{j}\right\}$. Let $\bar{j}$ be the smallest $j$ for which $A_{j} \neq 0$. We claim that $\rho_{\bar{j}}>1$. Note that $\rho_{j}=1$ only if $12 \mid d$. Indeed $\sqrt{2\left(1-\cos \left(\frac{2 \pi i}{d}\right)\right.}=1$ if $\cos \left(\frac{2 \pi i}{d}\right)=\frac{1}{2}$, i.e. if $\frac{2 \pi i}{d}=\frac{\pi}{3}$, or $\frac{2 \pi i}{d}=\frac{5 \pi}{3}$. The latter happens if $i=\frac{d}{6}$, and $i=\frac{5 d}{12}$ correspondingly, i.e. if $6 \mid d$ or $12 \mid d$ respectively.

Suppose on the contrary that $\Lambda_{n}^{*}=\sum_{j \geq \tilde{j}} A_{j} \rho_{j}^{n}$ with $\rho_{\tilde{j}}=1, A_{j}=0$ for all $j<\tilde{j}$, and $\rho_{j} \leq 1$ for all $j \geq \tilde{j}$. Assume first that $A_{\tilde{j}}=0$. Then $\Lambda_{n}^{*}=\sum_{j \geq \tilde{j}+s} \rho_{j}^{n} A_{j}$, where $A_{\tilde{j}+s}, \tilde{j}+s=\bar{j}, s>0$, is a smallest index for which $A_{j} \neq 0$. But then $\Lambda_{n}^{*}=\rho_{\tilde{j}+s}^{n}\left(A_{\tilde{j}+s}+\ldots\right)$, where the remaining terms consist of powers of reals $\frac{\rho_{\tilde{j}}}{\rho_{\tilde{j}+s}}<1$. The conditions $\Lambda_{n}^{*} \rightarrow 0$ and $\Lambda_{n}^{*} \in \mathbb{Z}$ show that $A_{\tilde{j}+s}=0$. Repeating the argument we get $A_{\tilde{j}+s}=A_{\tilde{j}+s+1}=\cdots=A_{r}=0$, i.e. $A_{j}=0$ for all $j$. It gives that $\Lambda_{n}^{*}=0$ for every $n \geq 1$. Assume next that $A_{\tilde{j}} \neq 0$. Then by the same argument as above $A_{\tilde{j}+s}=0$ for $\tilde{j}<\tilde{j}+s \leq r$, because

$$
\mathbb{Z} \ni \Lambda_{n}^{*}-A_{\bar{j}}=\sum_{j>\tilde{j}} A_{j} \rho_{j}^{n} \rightarrow 0 .
$$


This shows that $\Lambda_{n}^{*}=A_{\bar{j}}$ is a constant non-zero sequence. This is impossible with respect to the following inversion formula (see Wi], p. 125).

Lemma 2.10. If $\Lambda_{n}$ and $\Lambda_{n}^{*}$ are two integral sequences (arithmetic functions), and

$$
\Lambda_{n}^{*}=\sum_{j=0}^{n}(-1)^{n-j}\left(\begin{array}{c}
n \\
j
\end{array}\right) \Lambda_{j}, \quad \text { then } \quad \Lambda_{n}=\sum_{j=0}^{n}\left(\begin{array}{c}
n \\
j
\end{array}\right) \Lambda_{j}^{*},
$$

and conversely.

To complete the proof of Proposition 2.9, if $A_{j}=0$ a for all $1 \leq j \leq r$, then $\Lambda_{n}^{*}=0$ for every $n \geq 1$ and $\Lambda_{n}=\Lambda_{0}$ for every $n \geq 1$ by Lemma 2.10. contrary to our assumption on $\Lambda_{n}$. If $\bar{j}$ is the smallest index such that $A_{j} \neq 0$ and $\rho_{\bar{j}} \leq 1$, then $\rho_{\bar{j}}=1$ and $\Lambda_{n}^{*}=A_{\bar{j}}$ for every $n \geq 1$ as was shown above. By Lemma [2.10, then $\left|\Lambda_{n}\right| \rightarrow \infty$, contrary to our assumption on $\Lambda_{n}$. This shows that $\rho_{\bar{j}}>1$.

Consequently, for $n=2 l k, k \in \mathbb{N}$, the sum expressing $\Lambda_{n}^{*}$ has the asymptotic behavior as $A_{\bar{j}} \rho_{\bar{j}}^{n}$ with $\rho_{\bar{j}}>1$, where $1 \leq \bar{j} \leq r$ is the smallest index for which $A_{j}=\sum_{(d, i) \in R_{j}} a_{d} \neq 0$.

Remark 2.11. Note that in the case $l=2$, i.e. the sequence $\left\{\Lambda_{n}\right\}$ is 2-periodic, the supposition that $\left\{\Lambda_{n}\right\}$ is not a constant sequence is equivalent to $a_{2} \neq 0$. But the latter leads to the first considered case, and consequently

$$
\Lambda_{n}^{*}=a_{2} 2^{2 k}+a_{1}=2^{2 k}\left(a_{2}+\frac{a_{1}}{2^{2 k}}\right) .
$$

\section{A RATE OF GROWTH OF PERIOdic SOlutions}

We will show that Theorems 2.1 and 2.9 provide information about the rate of growth of the number of periodic solutions as a function of the minimal period. This result can be derived by an analysis of the rate of growth of periodic solutions of the associated Poincaré map.

We emphasize that it is possible to estimate from below the number of $n T$ periodic orbits, i.e. orbits for which $n T$ is a period (not necessarily minimal). This is a consequence of a corresponding theorem of Babienko and Bogatyi estimating from below the number of periodic orbits of a smooth map $f$ (cf. [BB]), with an estimate which does not depend on $f$ in the case where the cohomology ring of the space has a special form (cf. G] ). More precisely, let us denote by $\# \operatorname{Or}(\Phi, n)$ the cardinality of periodic orbits of $\Phi_{(0, T)}$ up to the length $n$, and by

$$
\#\{n T-\text { per sol }\}
$$

the cardinality of the set of all $n T$-periodic solutions for which $n T$ is the minimal period.

Proposition 3.1. Suppose as in Theorem 2.1 that $W$ is a periodic segment for the local process generated by (11), and the sequence $\left\{\Lambda_{n}\right\}$ is unbounded. Then there exists $n_{0}=n_{0}(\Phi)$ such that for every $n \geq n_{0}$,

$$
\sum_{k \mid n} \#\{k T-\text { per sol }\}=\# \operatorname{Or}(\Phi, n) \geq \frac{n}{D \cdot 2^{[(m+1) / 2]}}-\frac{n_{0}}{D \cdot 2^{[(m+1) / 2]}},
$$

where $D=\operatorname{dim}_{\mathbb{R}} H_{*}\left(W_{0}, W_{0}^{--}\right)$and $m$ is the dimension of $M$. 
Proof. First note that there is a one-one correspondence between the orbits of $\Phi_{(0, t)}$ of length $k$ (i.e. $k$ is the minimal period of any point of this orbit) and $k T$-periodic solutions of (11) of minimal period $k T$. Indeed, each two $k$-periodic points $x, y$ in one orbit of $\Phi_{(0, T)}$, i.e. $\Phi_{(0, T)}^{q}(x)=y, q \mid k$, induce one $n T$-periodic solution of (11). Surely, two $n$-periodic points lying in different orbits of $\Phi_{(0, T)}$ give different $n T$-solutions. Thus, we have that

$$
\sum_{k \mid n} \#\{k T-\text { per sol }\}=\sum_{k \mid n} \frac{\# P_{k}(\Phi)}{k}=\# \operatorname{Or}(\Phi, n) .
$$

Now we claim that

$$
\# \operatorname{Or}(\Phi, n) \geq \frac{\left(n-n_{0}\right)}{D \cdot 2^{[(m+1) / 2]}} .
$$

Indeed one can check that all the arguments of the proof of a corresponding theorem of Babenko and Bogatyi for a $C^{1}$-map of a compact closed manifold of dimension $m$ (cf. $\left[\mathrm{BB}\right.$ or $[\mathrm{JM}]$, Th. 3.2.41) carry over to this case. Here $\Phi_{(0, T)}$ is a $C^{k}$ map, $k \geq 1$, of the compact pair $\left(W_{0}, W_{0}^{--}\right)$, but the fixed point index $\operatorname{ind}\left(\Phi_{(0, T)}^{n}\right)$ is equal to $\Lambda_{n}$, the Lefschetz number of $n$-th iteration of a map of pairs of compact ENR's, which gives control over the behavior of the sequence $\left\{\operatorname{ind}\left(\Phi_{(0, T)}^{n}\right)\right\}_{1}^{\infty}$.

In the second case we have to use another argument to estimate the rate of growth of $n T$-periodic solutions of equation (11) with respect to $n$. We are able to show an exponential growth of $n T$-periodic solutions for this case.

For $n \geq 1$ we denote by $P_{n}(\Phi)$ the set of all $n$-periodic points of the Poincaré map $\Phi_{(0, T)}$ with minimal period $n$, i.e.,

$$
P_{n}(\Phi)=\left\{x \in M: \Phi_{(0, T)}^{n}(x)=x, n \text { is minimal period of } x\right\},
$$

and respectively by $P^{n}(\Phi)$ the set of all $n$-periodic points the Poincaré map $\Phi_{(0, T)}$, i.e.

$$
P^{n}(\Phi)=\operatorname{Fix}\left(\Phi_{(0, T)}^{n}\right)=\bigcup_{k \mid n} P_{k}(\Phi) .
$$

Let $\# P_{n}(\Phi)$, correspondingly $\# P^{n}(\Phi)$, be the cardinality of $P_{n}(\Phi)$, respectively of $P^{n}(\Phi)$.

Proposition 3.2. Assume as in Theorem 2.1 that periodic segments $Z, W$ for equation (11) satisfy (5)-(6), and the sequence $\left\{\Lambda_{n}\right\}_{1}^{\infty}$ is bounded but not constant. Let $l, k_{0}$ be natural numbers as in Proposition [2.9,

Then for every $n=2 l k q, k, q \in \mathbb{N}, k>k_{0}$ and $q$ even we have

$$
\# P^{n}(\Phi) \geq \frac{2^{n}}{2 l k}-1
$$

Consequently, for every such $n$ we have

$$
\sum_{m \mid n} \#\{m T-\text { per sol }\} \geq \frac{\# P^{n}(\Phi)}{n} \geq \frac{1}{n}\left(\frac{2^{n}}{2 l k}-1\right) .
$$

Proof. For each natural number $n$ we define

$$
S(n)=\left\{s \in \mathbb{N}: \Lambda_{s}^{*} \neq 0, s \leq n\right\} .
$$

For $s \in S(n), \Lambda_{s}^{*} \neq 0$, hence by the formula (9) we obtain that for each $n$-periodic sequence $c \in \Sigma_{2}$ such that the symbol 1 appears $s$ times in $c$, there exists $x_{c} \in g^{-1}(c)$ 
and $\Phi_{(0, T)}^{n}\left(x_{c}\right)=x_{c}$. We put $m=2 l k$. From Proposition 2.9 it follows that $m d \in S(n)$ for $d=1, \ldots, q$, so for $n=m q$ we have

$$
\# P^{n}(\Phi) \geq \sum_{j \in S(n)}\left(\begin{array}{c}
n \\
j
\end{array}\right) \geq \sum_{d=1}^{q}\left(\begin{array}{c}
n \\
m d
\end{array}\right)=\sum_{j \geq 0, m \mid j}^{n}\left(\begin{array}{l}
n \\
j
\end{array}\right)-1 \geq \frac{2^{n}}{m}-1,
$$

by Lemma 3.3. This shows the first part of the proposition. The second part follows from the first by the same argument as in Proposition 3.1, since

$$
\# \sum_{k \mid n}\{\mathrm{kT}-\text { per sol }\}=\# \operatorname{Or}(\Phi, n)=\sum_{k \mid n} \frac{\# P_{k}(\Phi)}{k} \geq \frac{\sum_{k \mid n} \# P_{k}(\Phi)}{n}=\frac{\# P^{n}(\Phi)}{n} \text {. }
$$

Lemma 3.3. Let $n \in \mathbb{N}$ and $m \mid n$ be its divisor. Then

$$
\sum_{j \geq 0, m \mid j}^{n}\left(\begin{array}{l}
n \\
j
\end{array}\right)=\frac{2^{n}}{m}+\frac{2^{n}}{m}\left(\sum_{r=1}^{m-1}(-1)^{\frac{r n}{m}} \cos ^{n}\left(\frac{\pi r}{m}\right)\right) .
$$

In particular, if $n$ and $\frac{n}{m}$ are even, then

$$
\sum_{j \geq 0, m \mid j}^{n}\left(\begin{array}{l}
n \\
j
\end{array}\right) \geq \frac{2^{n}}{m}
$$

Proof. We observe first that for $\omega=\exp \left(\frac{2 \pi \imath}{m}\right)$

$$
\sum_{j \geq 0, m \mid j}^{n}\left(\begin{array}{l}
n \\
j
\end{array}\right)=\frac{1}{m} \sum_{r=0}^{m-1}\left(1+\omega^{r}\right)^{n}
$$

Indeed, since

$$
\sum_{r=0}^{m-1} \omega^{l j}= \begin{cases}m, & \text { if } m \mid j \\ 0, & \text { otherwise }\end{cases}
$$

we get

$$
\sum_{j \geq 0, m \mid j}^{n}\left(\begin{array}{l}
n \\
j
\end{array}\right)=\sum_{j=0}^{n} \frac{1}{m} \sum_{r=0}^{m-1} \omega^{r j}\left(\begin{array}{l}
n \\
j
\end{array}\right)=\frac{1}{m} \sum_{r=0}^{m-1} \sum_{j=0}^{n}\left(\begin{array}{l}
n \\
j
\end{array}\right)\left(\omega^{r}\right)^{j}=\frac{1}{m} \sum_{r=0}^{m-1}\left(1+\omega^{r}\right)^{n} .
$$

Since $m \mid n$, it follows that

$$
\begin{gathered}
\sum_{j \geq 0, m \mid j}^{n}\left(\begin{array}{l}
n \\
j
\end{array}\right)=\frac{1}{m} \sum_{r=0}^{m-1}\left(1+\omega^{r}\right)^{n}=\frac{2^{n}}{m}+\frac{2^{n}}{m} \sum_{r=1}^{m-1}\left(\frac{1+\omega^{r}}{2}\right)^{n} \\
=\frac{2^{n}}{m}+\frac{2^{n}}{m} \sum_{r=1}^{m-1} \cos ^{n}\left(\frac{\pi r}{m}\right) \cdot \exp \left(\frac{r n \pi \imath}{m}\right)=\frac{2^{n}}{m}+\frac{2^{n}}{m} \sum_{r=1}^{m-1} \cos ^{n}\left(\frac{\pi r}{m}\right) \cdot(-1)^{\frac{r n}{m}} .
\end{gathered}
$$

Remark 3.4. The simplest case of non-constant $m+1$-periodic sequences $\Lambda_{n}$ such that

$$
\Lambda_{1}=\ldots=\Lambda_{m}, \quad \Lambda_{0} \neq \Lambda_{1},
$$

was studied in $\mathrm{W}, \mathrm{PW}, \mathrm{SW}, \mathrm{SWZ}$ in order to detect chaotic behavior in the planar equations

$$
z^{\prime}=\left(1+e^{i \kappa t}|z|^{2}\right) \bar{z}^{m} .
$$


It follows (compare [PW, $[\mathrm{W}]$ ) that in this case for $k>0, \Lambda_{k}^{*}=0$ if and only if $m$ is odd and $k$ is the odd multiplicity of $m$, we are able to get a better estimation for the growth of the sequence $\Lambda_{k}^{*} \neq 0$. In particular, if $m$ is even, then $\Lambda_{k}^{*} \neq 0$ for each $k$, so by a modification of the arguments in the proof of the above result, for each prime $p$ we get

$$
\# P_{p}(\Phi) \geq \sum_{k=1}^{p-1}\left(\begin{array}{l}
p \\
k
\end{array}\right)=2^{p}-2 .
$$

Proposition 3.5. Assume that periodic segments $Z, W$ for equation (1) satisfy (5) -(6), and the sequence $\left\{\Lambda_{n}\right\}_{0}^{\infty}$ is such that $\Lambda_{1} \neq \Lambda_{0}$. If $p>\left|\Lambda_{1}-\Lambda_{0}\right|$ is an odd prime, then

$$
\# P_{2 p}(\Phi) \geq \frac{4^{p}}{p}-4
$$

Proof. By Lemma 2.6 the dual sequence $\Lambda_{n}^{*}$ satisfies Dold's relations, so we get

$$
\Lambda_{p}^{*} \cong \Lambda_{1}^{*} \bmod p .
$$

Since $\Lambda_{1}^{*}=\Lambda_{1}-\Lambda_{0}$, we have

$$
\Lambda_{p}^{*} \not 0 \quad \bmod p
$$

and consequently $\Lambda_{p}^{*} \neq 0$. Again, by formula (9) we obtain that for each $2 p$-periodic sequence $c=\left(c_{0}, \ldots, c_{2 p-1}\right) \in \Sigma_{2}$ such that the symbol 1 appears $p$ times in $c$, there exists $x_{c} \in g^{-1}(c)$ and $\Phi_{(0, T)}^{2 p}\left(x_{c}\right)=x_{c}$. Let us observe that if $2 p$ is a minimal period of the sequence $c$, then $x_{c} \in P_{2 p}(\Phi)$. Since $p$ is prime, the minimal period $\tau_{c}$ of the sequence $c$ belongs to the set $\{1,2, p, 2 p\}$. Since there are exactly $p$ symbols 1 in $c$, then $\tau_{c} \neq 1$. Similarly, since $p$ is odd, then $\tau_{c} \neq p$. Otherwise, the symbol 1 will appear in $c$ an even number of times. On the other hand, there are exactly two $2 p$ periodic sequences $c$ with $p$ symbols 1 and minimal period $\tau_{c}=2$. It follows that $\tau_{c} \in\{2,2 p\}$ and consequently

$$
\# P_{2 p}(\Phi) \geq\left(\begin{array}{c}
2 p \\
p
\end{array}\right)-2
$$

The proof is finished since by Lemma 3.3 with $n=2 p, m=p$

$$
\left(\begin{array}{c}
2 p \\
p
\end{array}\right)=\sum_{k \in\{0,1,2\}}\left(\begin{array}{l}
2 p \\
k p
\end{array}\right)-2 \geq \frac{2^{2 p}}{p}-2
$$

\section{REFERENCES}

[BB] I. K. Babienko, S. A. Bogatyi, Behaviour of the index of periodic points under iterations of mapping. Izv.-Akad.-Nauk-SSSR-Ser.-Mat., 55 No. 1, (1991), 3-31.

[D] A. Dold, Fixed point indices of iterated maps. Invent. Math., 74, (1983), 419-435. MR0724012 (85c:54077)

[G] G. Graff, Minimal periods of maps of rational exterior spaces, Fundamenta Math., 163, (2000), 99-115. MR.1752098(2001d:37018)

[JM] J. Jezierski, W. Marzantowicz, Homotopy Methods in Topological Fixed and Periodic Point Theory. Series: Topological Fixed Point Theory and Its Applications, Vol. 3, Springer, (2005), XI, 319 p., ISBN: 1-4020-3930-1. MR2189944 (2006i:55003)

[MP] W. Marzantowicz, P. M. Przygodzki, Finding periodic points of a map by use of a $k$-adic expansion. Discrete and Cont. Dyn. Sys., vol 5, (1999), 495-514. MR.1696325 (2000c:37024)

[PW] L. Pieniążek, K. Wójcik, Complicated dynamics in non-autonomous ODE's. Universitatis Iagellonicae Acta Mathematica XLI, (2003), 163-179. MR2084760 (2005g:34101) 
[ShSu] M. Shub and P. Sullivan, A remark on the Lefschetz fixed point formula for differentiable maps, Topology, 13, (1974), 189-191. MR0350782 (50:3274)

[S1] R. Srzednicki, Periodic and bounded solutions in blocks for time-periodic nonautonomous ordinary differential equations, Nonlinear Anal. 22, (1994), 707-737. MR1270166 (95c:34076)

[S2] R. Srzednicki, Ważewski method and the Conley index. in Handbook of Differential Equations vol 1, Edited by A. Canada, P. Drabek, A. Fonda, 591-684. MR2166495 (2006j:37014)

[SW] R. Srzednicki, K. Wójcik, A geometric method for detecting chaotic dynamics., J. Diff. Eq., 135, (1997), 66-82. MR1434915 (98f:58140)

[SWZ] R. Srzednicki, K. Wójcik, P. Zgliczyński, Fixed point results based on Ważewski method. in "Handbook of topological fixed point theory" Ed: R. Brown, M. Furi, L. Górniewicz, B. Jiang, 903-941, Springer, Dordrecht, (2005). MR2171125 (2006g:37015)

[Su] Zhi-Wei Sun, Combinatorial identities in dual sequences. European J. Combin. 24, (2003), no.6, 709-718. MR1995582 (2004g:05017)

[Wi] H. S. Wilf, Generatingfunctionology (2nd edition), Academic Press, 1994. MR:1277813 (95a:05002)

[W] K. Wójcik, On detecting periodic solutions and chaos in the time periodically forced ODE's. Nonlinear Anal. TMA 45, (2001), 19-27. MR.1828064(2003b:34092)

Faculty of Mathematics and Computer Science, Adam Mickiewicz Universiy of Poznań, Umultowska 67, 61-614 Poznań, Poland

PWSZ Nowy Saç, Institute of Pedagogy, Ul. Chruślicka 6, 33-300 Nowy Saç, Poland and Institute of Mathematics, Jagiellonian University, Reymonta 4, 30-059 Kraków, POLAND 